\title{
Fault Diagnosis of an Advanced Wind Turbine Benchmark Using Interval-Based ARRs and Observers
}

\author{
Hector Sanchez, Teresa Escobet, Vicenç Puig and Peter Fogh Odgaard, Senior Member, IEEE
}

\begin{abstract}
This paper proposes a model-based fault diagnosis approach for wind turbines and its application to a realistic wind turbine fault diagnosis benchmark. The proposed fault diagnosis approach combines the use of analytical redundancy relations (ARRs) and interval observers. Interval observers consider an unknown but bounded description of the model parametric uncertainty and noise using the the so-called set-membership approach. This approach leads to formulate the fault detection test by means of checking if the measurements fall inside the estimated output interval, obtained from the mathematical model of the wind turbine and noise/parameter uncertainty bounds. Fault isolation is based on considering a set of ARRs obtained from structural analysis of the wind turbine model and a fault signature matrix that considers the relation of ARRs and faults. The proposed fault diagnosis approach has been validated on a 5MW wind turbine using the NREL FAST simulator. The obtained results are presented and compared with other approaches proposed in the literature.
\end{abstract}

Index Terms-Analytical redundant relations, interval-based observers, model-based fault diagnosis, wind turbines.

\section{INTRODUCTION}

Wind turbines have become an important source of renewable power generation in the last years. A major issue with wind turbines systems specially those located offshore, is the relatively high cost of operation and maintenance (OM). Also, poor reliability directly reduces availability of wind power due to the turbine downtime [1].

Fault diagnosis (FD) and fault tolerant control (FTC) of wind turbines is an important issue in order to decrease the operation and maintenance costs and increase penetration into electrical grids because the enhanced reliability those techniques can provide. However, the complexity of large modern wind turbines makes difficult to transfer advanced FD and FTC methods from the theory to application.

Manuscript received March 31, 2014; revised April 13, 2014, April 16, 2014, June 28, 2014, September 11, 2014 and November 5, 2014; accepted November 12, 2014.

Copyright (c) 2015 IEEE. Personal use of this material is permitted. However, permission to use this material for any other purposes must be obtained from the IEEE by sending a request to pubs-permissions@ieee.org.

This work has been funded by the Spanish MINECO through the project CYCYT SHERECS (ref. DPI2011-26243) and the grant BES-2012-058489.

H. Sanchez, T. Escobet and V. Puig are with the Automatic Control Department, Universitat Politècnica de Catalunya (UPC), Rambla Sant Nebridi 22, 08222 Terrassa, Spain; (e-mail:\{hector.eloy.sanchez, teresa.escobet, vicenc.puig\}@upc.edu).

P.F. Odgaard is with the Department of Electronic Systems, Automation and Control Section, Aalborg University, Fredrik Bajers Vej 7C, 9220 Aalborg, Denmark; (e-mail:pfo@es.aau.dk).
As discussed in [2], in the wind turbine industry, Condition Monitoring Systems (CMS) are commonly used for early detection of faults in order to minimize downtime and maximize productivity. CMS comprises a set of sensors and signal processing equipment/algorithms that provide continuous indications of component (and, consequently wind turbine) condition. There have been a few literature reviews on wind turbine condition monitoring such as [2].

As the wind energy have gained increasing attention from industries and academia since 2006, many new research works have been reported towards integrating advanced fault diagnosis algorithms in the CMS. Currently, most of the CMS in wind turbines are data-based and utilize e.g. vibration and acoustic emission measurements [3], frequency domain analysis [4], time domain analysis [5] and support vector machines [6]. Only recently, some work has been done on model-based fault diagnosis in wind turbines. For example, an observer based scheme to detect sensor faults in the pitch system was presented in [7] and a parity equations based scheme for fault detection in wind turbines was proposed in [8].

Some of the wind turbine components are more likely to fail or have a higher cost than others, and because of this reason many works found in the literature are focused in some of the components rather than others. Some works for the following components were found in the literature: gearbox [5] [9], bearings [10], pitch system [11] and electrical system $[8,12,13,14,15,16]$.

The main contribution of this paper is to propose a modelbased fault diagnosis approach for wind turbines and its application to a realistic wind turbine FD and FTC benchmark proposed in [17]. The proposed fault diagnosis approach combines the use of analytical redundancy relations (ARRs) and interval observers. Interval observers consider an unknown but bounded description of the model parametric uncertainty and noise using the so-called set-membership approach [18]. This approach leads to formulate the fault detection test by means of checking if the measurements fall inside the estimated output interval, obtained from the mathematical model of the wind turbine and noise/parameter uncertainty bounds. Fault isolation is based on considering a set of ARRs obtained from structural analysis of the wind turbine model and a fault signature matrix that considers the relation of ARRs and faults. Finally, the proposed fault diagnosis approach has been validated on a 5MW wind turbine using the NREL FAST simulator. The obtained results are presented and compared with other approaches proposed in the literature. 
The structure of the paper is the following: In Section II, an overview of the wind turbine benchmark is presented. Section III presents the ARRs obtained from the available measurements and the considered FD wind turbine models using the structural analysis. Section IV presents the fault detection scheme based on interval models and how parametric uncertainty is estimated. The fault diagnosis scheme is presented in Section V. The results obtained with the proposed approach applied to the advance wind turbine benchmark are summarized in Section VI and in Section VII are compared with other approaches reported in the literature. Conclusions are presented in Section VIII.

\section{Overview of THE Wind TuRbine Benchmark}

As discussed in the introduction, the growing interest in wind turbines, coming from both the academia and the industry, motivated the proposal of a wind turbine benchmark for FD and FTC, containing the most common faults reported in practice (see [19]). This benchmark was based on a realistic generic three blade horizontal variable speed wind turbine with a full converter coupling. In the Spring of 2010, kk-electronic together with other partners (MathWorks and Aalborg University) released an international competition on Fault Detection and Isolation (FDI) in Wind Turbines based on this benchmark model of the wind turbine. The competition consisted on finding the best schemes to diagnose and handle the different faults proposed and the results were presented in [19].

After the announcement of results of the first benchmark, a second challenge was presented in [17] that differs from the previous challenge in the wind turbine model that is modeled using the aeroelastic FAST simulator. This simulator is able to consider the wind turbine flexible modes that are present in practice making FD more difficult when simple models neglecting these modes are used, as was the case in [19]. FAST simulator is designed and developed by U.S. National Renewable Energy Laboratory (NREL) [20], and is widely used in academia and industry making the FD results obtained more relevant regarding the real application. The simulator also has been certified after performing a comparative analysis with two different wind turbines and showing good results ${ }^{1}$. This second benchmark will be the one considered in this paper.

The benchmark wind turbine FAST model is based on a 5 MW three bladed variable speed wind turbine developed by NREL for scientific research ${ }^{2}$. This model has been used to establish the reference specifications for a number of research projects supported by the U.S. DOEs Wind and Hydropower Technologies Program, the integrated European Union UpWind research program and the International Energy Agency (IEA). The NREL $5 \mathrm{MW}$ model has been used as a reference by many research teams throughout the world, to standardize baseline offshore wind turbine specifications and to quantify the benefits of advanced land and sea-based wind energy technologies. The turbine's hub height is $89.6 \mathrm{~m}$ and the rotor radius is $63 \mathrm{~m}$. The rated rotor speed is $12.1 \mathrm{rpm}$

\footnotetext{
${ }^{1}$ https://nwtc.nrel.gov/SimulatorCertification

${ }^{2}$ http://www.nrel.gov/docs/fy09osti/38060.pdf
}

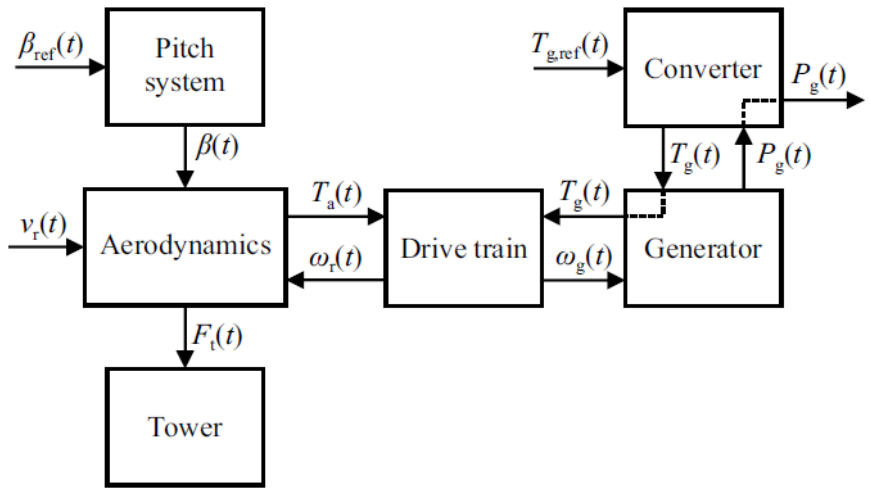

Fig. 1. Subsystems models interaction of the wind turbine system model.

while the generator speed is $1200 \mathrm{rpm}$. The simulator also includes baseline controllers that allow to control the three pitch angles, generator and converter torques and yaw position. Different measurements are available from sensors as well as the control references. Table I shows the different sensors provided in the benchmark model with the measurement noise modeled as a Gaussian white noise and considering a sampling period of $T_{s}=0.0125 \mathrm{~s}$.

TABLE I

BENCHMARK AVAILABLE SENSORS

\begin{tabular}{|l|c|c|c|}
\hline Sensor Type & Symbol & Unit & Noise \\
\hline Anemometer & $v_{w, m}$ & $\mathrm{~m} / \mathrm{s}$ & 0.0071 \\
\hline Rotor Speed & $\omega_{r, m}$ & $\mathrm{rad} / \mathrm{s}$ & $10^{-4}$ \\
\hline Generator Speed & $\omega_{g, m}$ & $\mathrm{rad} / \mathrm{s}$ & $2 \cdot 10^{-4}$ \\
\hline Generator Torque & $T_{g, m}$ & $\mathrm{Nm}$ & 0.9 \\
\hline Generated Electrical Power & $P_{g, m}$ & $\mathrm{~W}$ & 10 \\
\hline Pitch angle of $i$ th Blade & $\beta_{i, m}$ & $\mathrm{deg}$ & $1.5 \cdot 10^{-3}$ \\
\hline Blade root moment $i$ th blade & $M_{B, i, m}$ & $\mathrm{Nm}$ & $10^{3}$ \\
\hline Azimuth angle low speed side & $\phi_{m}$ & $\mathrm{rad}$ & $10^{-3}$ \\
\hline Yaw error & $\Xi_{e, m}$ & $\mathrm{deg}$ & $5 \cdot 10^{-2}$ \\
\hline Tower top acceleration (x, y directions) & $\begin{array}{l}\ddot{x}_{x, m} \\
\ddot{y}_{y, m}\end{array}$ & $\mathrm{~m} / \mathrm{s}^{2}$ & $5 \cdot 10^{-4}$ \\
\hline
\end{tabular}

A brief description of the wind turbine subsystems, illustrated in Figure 1, is presented below:

- Drive Train connects the low-speed shaft to the highspeed shaft increasing the rotational speed to a level required by the generator to produce electric energy.

- Generator/converter converts rotational energy into electric energy and adequate the electricity produced to the network.

- Pitch system is in charge of the blade pitching and it is used to maximize the efficiency in low winds. In high winds, it is used to control the aerodynamic torque and thereby control the rotor speed and tower blade loads.

- Tower supports the nacelle and the rotor. Since the wind speed increases with the height, a taller tower generally enables a wind turbine to generate more electric energy.

- Aerodynamics describes the transfer from the wind energy to rotational energy produced by the rotor.

Figure 2 presents a block diagram of the wind turbine simulation model, provided with the benchmark, including the 
feedback loops corresponding to the pitch, yaw and torque variables. In this figure, it also appears the components which are affected by the set of faults and the fault diagnosis block that will be designed in this paper.

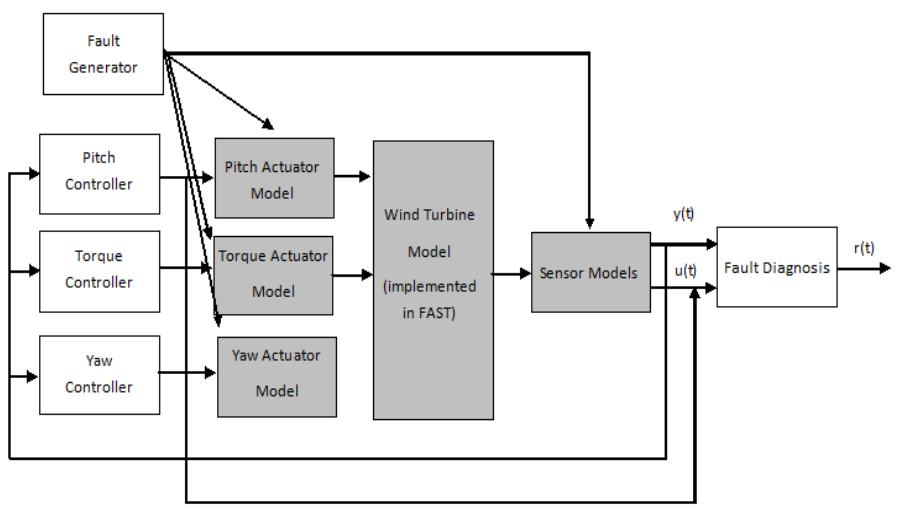

Fig. 2. Block diagram of wind turbine simulation model

The wind turbine benchmark defines a set of ten fault scenarios mainly introduced in sensors and actuators (see Fault Generator in Figure 2). The types of faults are offsets, scaling, stuck, changes in the system dynamics and bit errors, as shown in Table II. These faults are motivated by research in both public domain and proprietary sources [17].

TABLE II

FAULT SCENARIOS

\begin{tabular}{|c|l|l|}
\hline No. & Faults & Type \\
\hline$f_{1}$ & Blade root bending moment sensor & Scaling \\
\hline$f_{2}$ & Accelerometer & Offset \\
\hline$f_{3}$ & Generator speed sensor & Scaling \\
\hline$f_{4}$ & Pitch angle sensor & Stuck \\
\hline$f_{5}$ & Generator power sensor & Scaling \\
\hline$f_{6}$ & Low speed shaft position encoder & Bit error \\
\hline$f_{7}$ & Pitch actuator & Abrupt \\
\hline$f_{8}$ & Pitch actuator & Slow \\
\hline$f_{9}$ & Torque offset & Offset \\
\hline$f_{10}$ & Yaw drive & Stuck drive \\
\hline
\end{tabular}

\section{ARR GENERATION}

The design of the fault diagnosis system is based on deriving a set of ARRs by combining the model equations (associated to the subsystems presented in previous section) with the available sensors. This is the standard procedure to design a fault diagnosis system using model based approaches (for more details see [21]). Some of the model equations come directly from the benchmark model while the remaining are obtained using data-based techniques.

ARRs are defined as relations between known variables and can be derived combining the measurement model (known variables) with the system model (unknown variables). Combining the model equations with the available sensors (see Table I) described in the wind turbine benchmark [17], by means of the structural analysis approach and perfect matching algorithm [21], the resulting set of ARRs is presented in the following.

\section{A. Power System ARRs}

The generator and converter dynamics is modeled as in the benchmark [19] by a first-order model

$$
\frac{T_{g}(s)}{T_{g, r e f}(s)}=\frac{1}{\tau_{g} s+1}
$$

where $T_{g, r e f}$ is the reference for the generator torque $T_{g}$ and $\tau_{g}$ is the time constant of the first order model.

The power produced by the generator is given by

$$
P_{g}(t)=\eta_{g} \omega_{g}(t) T_{g}(t)
$$

where $P_{g}(t)$ is the power produced by the generator and $\eta_{g}$ is the efficiency of the generator.

$A R R l$ is obtained directly from the power equation presented in (2)

$$
P_{g, m}(t)=\eta_{g} \omega_{g, m}(t) T_{g, m}(t)
$$

since the power generated $P_{g, m}(t)$, the generator speed $\omega_{g, m}(t)$ and the generator torque $T_{g, m}(t)$ are all measured variables.

$A R R 2$ is obtained from the generator/converter model described in (1) as follows

$$
\tau_{g} \frac{d T_{g, m}(t)}{d t}+T_{g, m}(t)=T_{g, r e f}(t)
$$

\section{B. Blade Root Moment ARRs}

The modeling of the aerodynamics forces affecting the blades of the rotor is not an easy task. In the first attempts to model the blade root moment (BRM) dynamics, the model proposed in [22] was considered.

$$
\begin{aligned}
& M_{B, j}(t)=k_{1}\left(v_{r}(t)-\frac{d y(t)}{d t}+\frac{9 R_{b}}{8 H} \frac{d y(t)}{d t} \sin \left(\psi_{j}(t)\right)\right) \\
& +k_{2} \beta_{j}(t)
\end{aligned}
$$

where $M_{B, j}(t)$ and $\beta_{j}$ are the blade root moment and pitch angle of blade $j, \psi_{j}(t)$ is the azimuth angle of blade $j, v_{r}(t)$ is the wind speed, $\frac{d y(t)}{d t}$ is the translation speed of the nacelle from its equilibrium position, $R_{b}$ is the distance from the hub to the point where the thrust acts on the blade and $H$ is the hub height.

However, during the simulation tests performed with the benchmark based on the FAST simulator, some problems were found with this model and the behavior did not correspond to the one observed in the simulations. After analyzing the blade root moment behavior and considering the influence of the wind speed and blades pitch angle on this variable, an experimental model was proposed to be used. This experimental model is based on the mean values of the blade root moment and pitch angle signals in steady state to filter the flexible modes. Several tests with different constant wind speeds ranging from $12 \mathrm{~m} / \mathrm{s}$ to $30 \mathrm{~m} / \mathrm{s}$ were performed obtaining different pitch angle values. Then, a relation between the different pitch angles and the mean value of the blade root moment in steady state was found. As it can be seen in Figure 3 , first, second and third order polynomials were considered 


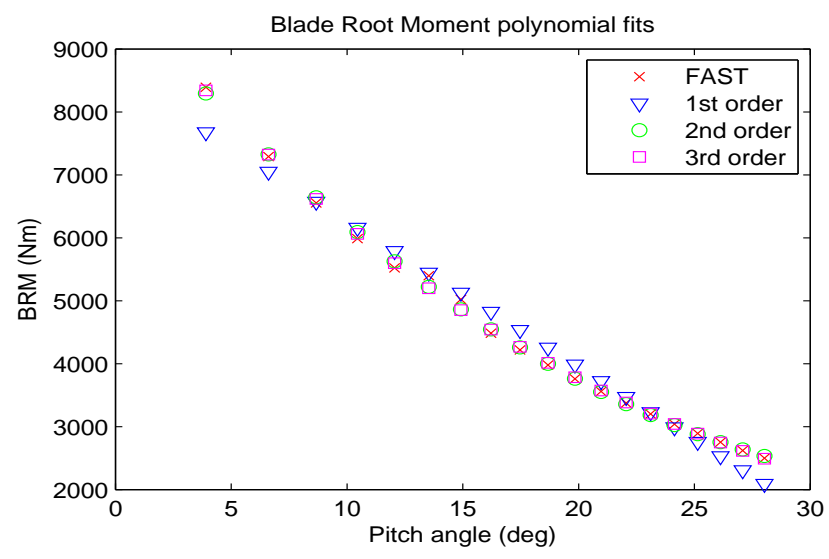

Fig. 3. Estimated Blade Root Moments models

to represent the mean blade root moment as function of the pitch angle.

Finally, the proposed model for the blade root moment dynamics was the third order blade root moment mean model

$$
\bar{M}_{B, j, m(t)}=a_{3} \beta_{j, m}(t)^{3}+a_{2} \beta_{j, m}(t)^{2}+a_{1} \beta_{j, m}(t)+a_{0}
$$

where: $\bar{M}_{B, j}(t)$ is the mean blade root moment and $\beta_{j}$ is the pitch angle on blade $j$. The values of the coefficients $a_{i}$ for blade root moment mean models for each blade, corresponding to experiments of winds higher than $12 \mathrm{~m} / \mathrm{s}$, are shown in Table III.

TABLE III

Blade Root Moment Mean Model Coefficients

\begin{tabular}{|c|c|c|c|c|}
\hline Mean BRM model & $a_{3}$ & $a_{2}$ & $a_{1}$ & $a_{0}$ \\
\hline$M_{B, 1(t)}$ & -0.0778 & 9.3204 & -469.4313 & 96482 \\
\hline$M_{B, 2(t)}$ & -0.0777 & 9.3159 & -469.3210 & 99720 \\
\hline$M_{B, 3(t)}$ & -0.0776 & 9.3228 & -469.6690 & 96495 \\
\hline
\end{tabular}

Thus, ARRs 3, 4 and 5 are obtained from the blade root moment model (6).

\section{Pitch System ARRs}

The hydraulic pitch system is modeled as a closed loop second order transfer function between the pitch angle $\beta$ and its reference $\beta_{r}$

$$
\frac{\beta(s)}{\beta_{r}(s)}=\frac{\omega_{n}^{2}}{s^{2}+2 \zeta \omega_{n} s+\omega_{n}^{2}}
$$

This model is the same used in [19].

ARRs 6, 7 and 8 are obtained from the pitch subsystems derived directly from the model (7)

$$
\frac{d^{2} \beta_{j, m}(t)}{d t^{2}}+2 \zeta \omega_{n} \frac{d \beta_{j, m}(t)}{d t}+\omega_{n}^{2} \beta_{j, m}(t)=\omega_{n}^{2} \beta_{r e f}(t)
$$

\section{Drive Train ARRs}

The drive train is modeled by a two mass and two shafts model that can be presented in input/output form as follows:

$$
\left\{\begin{array}{l}
\dot{\omega}_{r}(t)+\frac{1}{J_{r}}\left(B_{d t}+B_{r}\right) \omega_{r}(t)+\frac{K_{d t}}{J_{r}} \theta_{\Delta}(t)=\frac{B_{d t}}{J_{r} N_{g}} \omega_{g}(t) \\
+\frac{1}{J_{r}} T_{a}(t) \\
\dot{\omega}_{g}(t)+\frac{1}{J_{g}}\left(\frac{B_{d t}}{N_{g}^{2}}+\frac{B_{g}}{J_{g}}\right) \omega_{g}(t)-\frac{K_{d t}}{N_{g} J_{g}} \theta_{\Delta}(t)= \\
\frac{B_{d t}}{J_{g} N_{g}} \omega_{r}(t)-\frac{1}{J_{g}} T_{g}(t)
\end{array}\right.
$$

where: $B_{r}$ and $J_{r}$ are respectively the viscous friction and the moment of inertia of the low-speed shaft, $B_{g}$ and $J_{g}$ are the viscous friction and the moment of inertia of the high-speed shaft, $B_{d t}$ is the torsion damping coefficient of the drive train, $K_{d t}$ is the torsion stiffness of the drive train, $N_{g}$ is the gearbox ratio, $T_{g}(t)$ is the generator torque, $T_{a}(t)$ is the aerodynamic torque applied to the rotor, $\omega_{g}(t)$ and $\omega_{r}(t)$ are the generator and rotor speed, respectively and $\theta_{\Delta}(t)$ is the torsion angle of the drive train.

From the drive train model (9), the following relations can be established in steady state that filter the flexible modes. First, a relation between $\omega_{g}(t)$ and $T_{g}(t)$ can be established as follows

$$
B_{g} \omega_{g}(t)=-T_{g}(t)+K
$$

where the constant $K$ is the torsion angle in steady state. In a similar way, a relation between $\omega_{g}(t)$ and $\omega_{r}(t)$ through $N_{g}$ can also be derived

$$
\omega_{g}(t)=N_{g} \omega_{r}(t)
$$

The aerodynamic torque is modeled as in [23]

$$
T_{a}(t)=\frac{1}{2 \omega_{r}(t)} \rho A v_{r}^{3}(t) C_{p}(\lambda(t), \beta(t))
$$

where: $C_{p}(\lambda(t), \beta(t))$ is the power coefficient, $\beta(t)$ is the pitch angle, $\lambda(t)$ is the tip-speed ratio, $A$ is the rotor swept area, $v_{r}(t)$ is the wind speed and $\rho$ is the air density, which is assumed to be constant.

$A R R 9$ and $A R R 10$ were derived from equations (11) and (10), as follows

$$
\begin{gathered}
B_{g} \omega_{g, m}(t)=-T_{g, m}(t)+K \\
\omega_{r, m}(t)-\frac{1}{N_{g}} \omega_{g, m}(t)=0
\end{gathered}
$$

Using the input/output equations of the drive train model presented in equation (9), with the dynamics of the torsion angle neglected because there is no sensor available for this variable in the benchmark, ARR 11 (eq. 15) and ARR 12 (eq. 16) are obtained

$$
\begin{aligned}
& f_{1}\left(\dot{\omega}_{r, m}(t), \omega_{r, m}(t), T_{a *}(t), T_{g, m}(t)\right)=0 \\
& f_{2}\left(\dot{\omega}_{g, m}(t), \omega_{g, m}(t), T_{a *}(t), T_{g, m}(t)\right)=0
\end{aligned}
$$

where the aerodynamic torque $T_{a *}(t)$ can be computed by the following expression

$$
T_{a *}(t)=f\left(\omega_{r, m}(t), v_{m}(t), \beta_{m}(t)\right)
$$

The rotor speed $\omega_{r}, m(t)$ and the azimuth angle $\phi_{m}(t)$ of the low speed shaft are both known variables. Therefore, the 
ARR 13 derived from (18) can be proposed as a relation between rotor speed and the derivative of the low speed shaft angle $\frac{d \phi_{m}(t)}{d t}$

$$
\omega_{r, m}(t)=\frac{d \phi_{m}(t)}{d t}
$$

\section{E. Yaw System ARRs}

ARR 14 is derived from the yaw controller model proposed in [17]. The controller has the measured yaw error $\Xi_{e, m}(t)$ as an input and the yaw reference angular velocity $\omega_{y, r}$ as an output, which are both known variables. The yaw rate is modeled as in [17] with a delay respect to the yaw reference angular velocity. Therefore, the following ARR can be obtained:

$$
f\left(\Xi_{e, m}(t), \omega_{y, r}(t)\right)=0
$$

\section{F. Tower System ARRs}

Wind tower deflects because of the loads and wind tower flexibility leading to the nacelle motion. This motion can be characterized by means of a deflection in wind direction (foreaft) $x_{t}$ and perpendicular to wind direction (sidewards) $y_{t}$. Some models in the literature were proposed such as [22]. However, in order to perform fault diagnosis using this tower model, a tower bottom strain gauge is needed. This sensor is not an industrial standard in wind turbines, and therefore is not included in the FD benchmark. To overcome the lack of this sensor, an experimental model with the purpose of fault diagnosis is proposed for the tower system. From the simulation tests performed with the benchmark based on FAST simulator, a relation between the tower top accelerations and the blade root moments in steady state was observed. The proposed model establishes an ARR containing the signals of the blade root moment and tower top acceleration in the $x$ direction (fore-aft), which are among the benchmark available sensors presented in Table II.

The proposed model is the following

$$
M_{B, j, m}(t)=a_{t_{1}} \ddot{x}_{x, m}(t)+b_{t_{1}}
$$

where $M_{B, j, m}(t)$ is the blade root moment on blade $j, \ddot{x}_{x, m}(t)$ is the accelerometer in the $x$ direction, $b_{t_{1}}$ is the mean value of $M_{B, j, m}(t)$ and $a_{t_{1}}$ is a parameter used to scale the acceleration signal.

The ARRs 15, 16 and 17 are obtained from the model (20).

\section{INTERVAL MODELS}

\section{A. Motivation}

The presence of flexible modes in the wind turbine (simulated with the aeroelastic FAST simulator) as discussed in Section II and the modeling errors inherent to the approximation of some model relations as discussed in Section III lead to the need of using a robust fault detection algorithm able to handle uncertainty [24]. One of the most developed families of approaches to deal with model uncertainty, called active, is based on generating residuals, which are insensitive to uncertainty (modeling errors and disturbances), while at the same time sensitive to faults using some decoupling method [24]. On the other hand, there is a second family of approaches, called passive, which enhances the robustness of the fault detection system at the decision-making stage using an adaptive threshold [25].

In this paper, the uncertainty will be located in the parameters bounding their values by intervals using the so-called interval models [25]. The robustness in fault detection is achieved by means of the passive approach at the decisionmaking stage using an adaptive threshold generated by considering the set of model responses obtained by varying the uncertain parameters within their intervals.

\section{B. Static and dynamic interval models}

ARRs introduced in Section III will be used to create residuals to detect and isolate faults. These residuals will be generated in order to check the consistency between the observed and the predicted process behavior. Looking at the obtained ARRs, they can be divided in two groups: static and dynamic. The static ARRs are: ARR $1, A R R 3, A R R 4$, $A R R 5, A R R 9, A R R 10, A R R 13$ and ARR14, while the remaining are dynamic.

The generation of residuals is straightforward in case of static ARRs since they follow directly from the mathematical expressions. On the other hand, in case of dynamic ARRs several options for generating residuals are possible ranging from parity equations to observers. In this paper, interval observers are used because they handle in a natural way the effect of the uncertainty by generating adaptive thresholds used for fault detection.

The model of each dynamic ARR is rewritten in observer canonical form as follows:

$$
\begin{aligned}
x(k+1) & =A(\tilde{\theta}) x(k)+B(\tilde{\theta}) u(k) \\
y(k) & =C(\tilde{\theta}) x(k)+\tilde{v}(k)
\end{aligned}
$$

where $u(k) \in \mathbb{R}^{n_{u}}$ is the system input, $y(k) \in \mathbb{R}^{n_{y}}$ is the system output, with $x(k) \in \mathbb{R}^{n_{x}}$ is the state-space vector, $\tilde{v}(k) \in \mathbb{R}^{n_{y}}$ is the output noise that is assumed to be bounded $\left|\tilde{v}_{i}(k)\right|<\sigma_{i}$, with $i=1, \ldots, n_{y}, A(\tilde{\theta}), B(\tilde{\theta}), C(\tilde{\theta})$, are matrices of appropriate dimensions where $\tilde{\theta} \in \mathbb{R}^{n_{\theta}}$ is the parameter vector. Uncertainty in the parameters is considered as follows

$$
\theta \in \Theta=\left\{\theta \in \mathbb{R}^{n_{\theta}} \mid \underline{\theta}_{i} \leq \theta_{i} \leq \bar{\theta}_{i}, i=1, \ldots, n_{\theta}\right\}
$$

Then, from the dynamic ARR expressed in state space form (21)-(22), a interval linear observer with Luenberger structure can be derived as follows [26]:

$$
\begin{aligned}
\hat{x}(k+1, \theta) & =(A(\theta)-L C(\theta)) \hat{x}(k, \theta)+B(\theta) u(k)+L y(k) \\
& =A_{0}(\theta) \hat{x}(k, \theta)+B(\theta) u(k)+L y(k) \\
\hat{y}(k, \theta) & =C(\theta) \hat{x}(k, \theta)
\end{aligned}
$$

where $\hat{x}(k, \theta)$ is the estimated system state vector, $\hat{y}(k, \theta)$ is the estimated system output vector and $A_{0}(\theta)=A(\theta)-L C(\theta)$ 
is the observer matrix. The observer gain matrix $L \in \mathbb{R}^{n_{x} \times n_{y}}$ is designed to stabilize the matrix $A_{0}(\theta)$ and to guarantee a desired performance regarding fault detection for all $\theta \in \Theta$ using the LMI pole placement approach [27].

The input/output form of the observer (24) is expressed as follows:

$$
\hat{y}(k, \theta)=G\left(q^{-1}, \theta\right) u(k)+H\left(q^{-1}, \theta\right) y(k)
$$

with:

$$
\begin{aligned}
G\left(q^{-1}, \theta\right) & =C(\theta)\left(q I-A_{0}(\theta)\right)^{-1} B(\theta) \\
H\left(q^{-1}, \theta\right) & =C(\theta)\left(q I-A_{0}(\theta)\right)^{-1} L
\end{aligned}
$$

The effect of the uncertain parameters $\theta$ on the observer temporal response $\hat{y}(k, \theta)$ will be bounded using an interval satisfying:

$$
\hat{y}(k, \theta) \in[\underline{\hat{y}}(k), \overline{\hat{y}}(k)]
$$

Such interval can be computed independently for each output $i=1, \ldots, n_{y}$, neglecting couplings among outputs, as follows:

$$
\underline{\hat{y}_{i}}(k)=\min _{\theta \in \Theta} \hat{y}_{i}(k, \theta) \quad \text { and } \quad \overline{\hat{y}}_{i}(k)=\max _{\theta \in \Theta} \hat{y}_{i}(k, \theta)
$$

subject to the observer equations given by (25). The optimization problems (29) could be solved using numerical methods as in [28] or, more efficiently by means of the zonotope approach presented in [29].

Finally, taking into account that the additive noise in the system (22) is bounded, the following condition should be satisfied

$$
y_{i}(k) \in\left[\underline{\hat{y}_{i}}(k)-\sigma_{i}, \overline{\hat{y}_{i}}(k)+\sigma_{i}\right] i=1, \ldots, n_{y}
$$

in a non-faulty scenario.

\section{Parameter uncertainty estimation}

One of the key points in passive robust model based fault detection is how models and their uncertainty bounds are obtained. Classical system identification methods are formulated under a statistical framework. Assuming that the measured variables are corrupted by additive noises with known statistical distributions and that the model structure is known, a parameter estimation algorithm will provide nominal values for the parameters together with descriptions of the associated uncertainty in terms of the covariance matrix or confidence regions for a given probability level [30]. However, this type of approaches cannot be applied when measurement errors are described as unknown but bounded values and/or modeling errors exist.

The problem of bounding the model uncertainty has been mainly stated in many references coming from the robust control field. For example, in [31], some methodologies that provide a model with its uncertainty have been developed, but always regarding its application to control. One of the methodologies for including uncertainty in the model assumes the bounded but unknown description of the noise and parametric uncertainty. This methodology is known as boundederror or set-membership estimation [32], which produces a set of parameters consistent with the selected model structure and the pre-specified noise bounds. This approach is used for estimating parametric uncertainty of the interval observers in (24).

The goal of the parameter estimation algorithm is to characterize the parameter set $\Theta$ (here a box) consistent with the data collected in a fault-free scenario. Given $N$ measurements of system inputs $y(k)$ and outputs $u(k)$ from a scenario free of faults and rich enough from the identifiability point of view, the parameter tolerance $\alpha$, and a nominal model described by a vector $\theta_{n}$ obtained using a standard least-square parameter estimation algorithm [33], the uncertain parameter estimation algorithm proceeds by solving the following optimization problem:

$\min \alpha$

subject to :

$$
\begin{aligned}
& y_{i}(k) \in\left[\underline{\hat{y}}_{i}(k)-\sigma_{i}, \overline{\hat{y}}_{i}(k)+\sigma_{i}\right] \quad i=1, \ldots, n_{y} \quad k=1, \ldots, N \\
& \underline{\hat{y}}_{i}(k)=\min _{\theta \in \Theta} \hat{y}_{i}(k, \theta) \quad i=1, \ldots, n_{y} \quad k=1, \ldots, N \\
& \overline{\hat{y}}_{i}(k)=\max _{\theta \in \Theta} \hat{y}_{i}(k, \theta) \quad i=1, \ldots, n_{y} \quad k=1, \ldots, N \\
& \hat{y}(k, \theta)=G\left(q^{-1}, \theta\right) u(k)+H\left(q^{-1}, \theta\right) y(k) \quad k=1, \ldots, N \\
& \Theta=\left[\theta_{n}(1-\alpha), \theta_{n}(1+\alpha)\right]
\end{aligned}
$$

Regarding the uncertain variables that appear in (30), it is assumed that a priori theoretical or practical considerations allow to obtain useful intervals associated to measurement noises, leading to an estimation of the noise bound $\sigma$. A similar algorithm can be used for the static ARRs.

\section{Fault Diagnosis Approach}

\section{A. Fault Detection}

Fault detection is based on generating a nominal residual comparing the measurements of physical system variables $y(k)$ with their estimation $\hat{y}(k)$ provided by the observer (24):

$$
r(k)=y(k)-\hat{y}\left(k, \theta_{n}\right)
$$

where $r(k) \in \mathbb{R}^{n_{y}}$ is the residual set and $\theta_{n}$ the nominal parameters. According to [34], the form of the nominal residual generator, obtained using (25), is:

$$
r(k)=\left(I-H\left(q^{-1}, \theta_{n}\right)\right) y(k)-G\left(q^{-1}, \theta_{n}\right) u(k)
$$

that has been derived taking into account the input/output form of the observer.

When considering model uncertainty located in parameters, the residual generated by (32) will not be zero, even in a nonfaulty scenario. To cope with the parameter uncertainty effect, a passive robust approach based on adaptive thresholding can be used [35], as previously discussed. Thus, using this approach, the effect of parameter uncertainty in the components $r_{i}(k)$ of residual $r(k)$ (associated to each system output $y_{i}(k)$ ) is bounded by the interval [28]:

$$
r_{i}(k) \in\left[\underline{r}_{i}(k)-\sigma_{i}, \bar{r}_{i}(k)+\sigma_{i}\right] \quad i=1, \ldots, n_{y}
$$

where:

$$
\underline{r}_{i}(k)=\underline{\hat{y}}_{i}(k)-\hat{y}_{i}\left(k, \theta_{n}\right) \text { and } \bar{r}_{i}(k)=\overline{\hat{y}_{i}}(k)-\hat{y}_{i}\left(k, \theta_{n}\right)
$$


The bounds $\hat{y}_{i}(k)$ and $\overline{\hat{y}}_{i}(k)$ of the system output estimation are computed component-wise using the interval observer (25). Then, the fault detection test is based on checking if the residuals satisfy or not the condition given by (34). In case that this condition does not hold, a fault can be indicated. Notice that checking condition (34) is equivalent to check condition (28).

As discussed in [26], fault detection based on interval observers presents non-detected faults (missed alarms) because of the uncertainty. This is due to the fact that there exists a minimum fault size that guarantees the activation of the fault detection test (34) despite the uncertainties. On the other hand, interval observers guarantee that there are no false alarms since uncertainty bounds are determined to explain all the data collected in non-faulty scenarios used for interval parameter estimation by means of (31).

\section{B. Fault Isolation}

Fault isolation consists in identifying the faults affecting the system. It is carried out on the basis of fault signatures, generated after the detection process, and their relation with all the considered faults. Robust residual evaluation presented in Section V-A allows obtaining a set of observed fault signatures $\phi(k)=\left[\phi_{1}(k), \phi_{2}(k), \ldots, \phi_{n r}(k)\right]$, where each fault indicator is given by:

$$
\phi_{i}(k)=\left\{\begin{array}{ccc}
0 & \text { if } & r_{i}(k) \in\left[\underline{r}_{i}(k)-\sigma_{i}, \bar{r}_{i}(k)+\sigma_{i}\right] \\
1 & \text { if } & r_{i}(k) \notin\left[\underline{r}_{i}(k)-\sigma_{i}, \bar{r}_{i}(k)+\sigma_{i}\right]
\end{array}\right.
$$

Standard fault isolation reasoning exploits the knowledge about the binary relation between the set of fault hypothesis and the set of residuals that is stored in the so called Fault Signature Matrix (FSM), denoted as $M$. An element $m_{n_{r}, n_{f}}$ ( $n_{r}$ indicates rows, $n_{f}$ indicates columns) of $M$ is equal to 1 if the fault $f_{n_{f}}$ affects the computation of the residual $r_{n_{r}}$; otherwise, the element $m_{n_{r}, n_{f}}$ is zero-valued. A column of $M$ is known as a theoretical fault signature and indicates which residuals are affected by a given fault. A set of faults is isolable if all the columns in $M$ are different (two columns that are equal indicate two faults that can not be distinguished).

Based on the use of FSMs, different reasoning procedures have been proposed in the literature, see for instance [36]. The procedure accepted as standard by the FDI community involves finding a matching between the observed fault signature and one of the theoretical fault signatures. However, this reasoning is not appropriate in an unknown but bounded context. Due to the uncertainty, when a fault is present in the system, an undefined number of the residuals affected by the fault can be found inconsistent, mainly depending on the sensitivity of each residual with respect to the fault and on the fault magnitude. In this case, if the columnmatching procedure is used, then the particular fault will not be identified. An appropriate reasoning which comes from the DX community only considers the residuals that are inconsistent when searching for the fault (that is, inconsistency is relevant, consistency is not). Based on the proposed framework by [36], the fault signature matrix is interpreted in DX CBD (Consistency-based Diagnosis) approach to fault isolation considering separately each line corresponding to a violated ARR, (i.e., a set of components that are to be considered abnormal in order to be consistent with the observed fault signature) before searching for a common explanation, i.e., follows a row view of the fault signature matrix.

Analyzing the effects of the faults presented in [17], listed in Table II, in the set of static and dynamic residuals obtained from the ARRs presented in Section III, the fault signature matrix shown in Table IV is obtained. This table shows the sensitivity of the obtained residuals in presence of the considered fault scenarios, where the sub-index $j$ takes the following values $j=1,2,3$.

TABLE IV

THEORETICAL SIGNATURE MATRIX

\begin{tabular}{|c|c|c|c|c|c|c|c|c|c|}
\hline & $f_{1-M_{\mathrm{B}, j, \mathrm{~m}}}$ & $f_{2}$ & $f_{3}$ & $f_{4}$ & $f_{5}$ & $f_{6}$ & $f_{(7-8)}$ & $f_{9}$ & $f_{10}$ \\
\hline $\mathrm{r}_{1}$ & & & $\mathrm{x}$ & & $\mathrm{x}$ & & & $\mathrm{x}$ & \\
\hline $\mathrm{r}_{2}$ & & & & & & & & $\mathrm{x}$ & \\
\hline $\mathrm{r}_{j+2}$ & $\mathrm{x}$ & $\mathrm{x}$ & & $\mathrm{x}$ & & & $\mathrm{x}$ & & \\
\hline $\mathrm{r}_{j+5}$ & & & & $\mathrm{x}$ & & & $\mathrm{x}$ & & \\
\hline $\mathrm{r}_{9}$ & & & $\mathrm{x}$ & & & & & $\mathrm{x}$ & \\
\hline $\mathrm{r}_{10}$ & & & $\mathrm{x}$ & & & & & & \\
\hline $\mathrm{r}_{11}$ & & & & & & & & $\mathrm{x}$ & \\
\hline $\mathrm{r}_{12}$ & & & $\mathrm{x}$ & & & & & $\mathrm{x}$ & \\
\hline $\mathrm{r}_{13}$ & & & & & & $\mathrm{x}$ & & & \\
\hline $\mathrm{r}_{14}$ & & & & & & & & & $\mathrm{x}$ \\
\hline $\mathrm{r}_{j+14}$ & $\mathrm{x}$ & $\mathrm{x}$ & & & & & & & \\
\hline
\end{tabular}

Based on the information of FSM presented in Table IV, the logical test that allows isolating the faults has been generated. Tables V and VI list the logical reasoning test in the case of applying column and row reasoning approaches respectively, where $\Delta N r_{i}$ indicates an abnormal behavior of $i^{\text {th }}$ residual.

TABLE V

COLUMN REASONING APPROACH

\begin{tabular}{|c|l|}
\hline Logical Test & Diagnostic \\
\hline$\Delta N r_{2+j}$ & $f_{1-M, B, j, m}$ \\
\hline$\Delta N r_{1} \wedge \Delta N r_{9} \wedge \Delta N r_{10} \wedge \Delta N r_{12}$ & $f_{3}$ \\
\hline$\Delta N r_{2+j} \wedge \Delta N r_{5+j}$ & $f_{4-\beta_{j}} \vee f_{(7-8)-P A_{j}}$ \\
\hline$\Delta N r_{1}$ & $f_{5}$ \\
\hline$\Delta N r_{1} \wedge \Delta N r_{2} \wedge \Delta N r_{9} \wedge \Delta N r_{11} \wedge \Delta N r_{12}$ & $f_{3}$ \\
\hline$\Delta N r_{13}$ & $f_{6}$ \\
\hline$\Delta N r_{14}$ & $f_{10}$ \\
\hline$\Delta N r_{16} \wedge \Delta N r_{17}$ & $f_{2}$ \\
\hline$\Delta N r_{15} \wedge \Delta$
\end{tabular}

TABLE VI

ROW REASONING APPROACH

\begin{tabular}{|c|l|}
\hline Logical Test & Diagnostic \\
\hline$\Delta N r_{1}$ & $f_{3} \vee f_{5} \vee f_{9}$ \\
\hline$\Delta N r_{2}$ & $f_{9}$ \\
\hline$\Delta N r_{2+j}$ & $f_{1-M, B, j, m} \vee f_{4-\beta_{j}} \vee f_{7-P A_{j}} \vee f_{8-P A_{j}}$ \\
\hline$\Delta N r_{5+j}$ & $f_{4-\beta j} \vee f_{7-P A_{j}} \vee f_{8-P A_{j}}$ \\
\hline$\Delta N r_{9}$ & $f_{3} \vee f_{9}$ \\
\hline$\Delta N r_{10}$ & $f_{3}$ \\
\hline$\Delta N r_{11}$ & $f_{9}$ \\
\hline$\Delta N r_{12}$ & $f_{3} \vee f_{9}$ \\
\hline$\Delta N r_{13}$ & $f_{6}$ \\
\hline$\Delta N r_{14}$ & $f_{10}$ \\
\hline$\Delta N r_{14+j}$ & $f_{1-M, B, j, m} \vee f_{2}$ \\
\hline
\end{tabular}

\section{RESULTS}

In the following, some representative results considering the fault scenarios defined in the wind turbine benchmark (see 
Section II) will be presented. The wind speed sequences used in the tests were some of the uniform mean wind speeds of $11 \mathrm{~m} / \mathrm{s}, 14 \mathrm{~m} / \mathrm{s}$ and $17 \mathrm{~m} / \mathrm{s}$, which are provided with the benchmark [17]. Additionally, a mean wind speed of $14 \mathrm{~m} / \mathrm{s}$ with direction changes to test the detection of fault $f_{10}$ in the yaw actuator was used.

In Figure 4, fault scenarios $f_{5}$ and $f_{2}$ are shown. Fault $f_{5}$ occurs in the generator power sensor scaled by a factor of 1.1 and it is present in the time interval from 240s to 265s. Fault scenario $f_{2}$ occurs in tower top accelerometer sensor in the $x$ direction corresponding to an offset of $-0.5 \mathrm{~m} / \mathrm{s}^{2}$ present in the time period from $75 \mathrm{~s}$ to $100 \mathrm{~s}$. All the faults defined in the benchmark [17] are only present during a determined period of time.
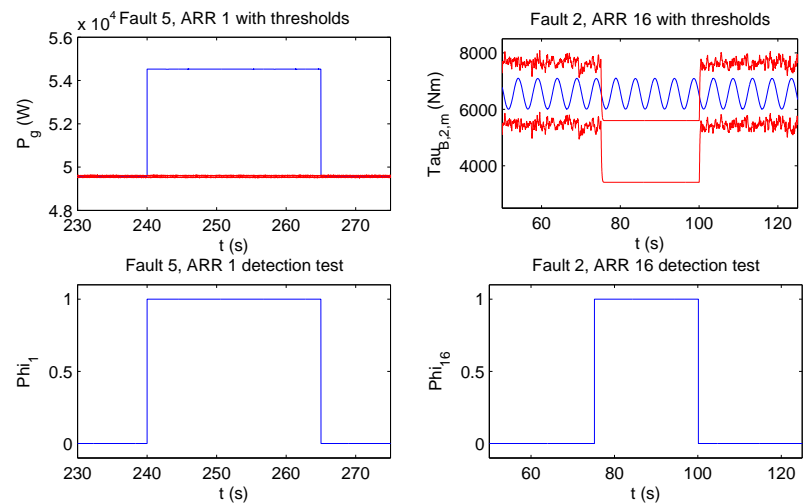

Fig. 4. Fault scenarios $f_{5}$ and $f_{2}$ : (up) measurement and detection thresholds and (down) fault indicator

In both fault scenarios shown in Fig 4, it can be observed that the measurement goes out of the detection thresholds and that the fault indicator activates during the interval of time in which the fault is present, either permanently or intermittently.

In Table VII, it is shown which residuals were activated for each one of the considered fault scenarios during the simulation tests. From this table, it can be observed that during the tests not all of the residuals are activated according to the FSM shown in Table IV.

TABLE VII

ACTIVATED RESIDUALS FOR EACH FAULT

\begin{tabular}{|c|c|}
\hline Fault No. & Activated Residuals \\
\hline$f_{1}$ & $r_{3}, r_{4}, r_{5}$ \\
\hline$f_{2}$ & $r_{15}, r_{16}$ and $r_{17}$ \\
\hline$f_{3}$ & $r_{1}, r_{9}$ and $r_{10}$ \\
\hline$f_{4}$ & $r_{3}, r_{4}, r_{5}, r_{6}, r_{7}$ and $r_{8}$ \\
\hline$f_{5}$ & $r_{1}$ \\
\hline$f_{6}$ & $r_{13}$ \\
\hline$f_{7}, f_{8}$ & $r_{3}, r_{4}$ and $r_{5}$ \\
\hline$f_{9}$ & $r_{2}$ and $r_{9}$ \\
\hline$f_{10}$ & $r_{14}$ \\
\hline
\end{tabular}

The fault detection results are resumed in Table VIII. The values for the required detection time $\left(t_{D}\right)$ are those specified in the benchmark. The real time detection $\left(t_{D}\right.$ real $)$ is the one obtained for the first residual activated in presence of the fault, where $T_{s}$ is the sampling period. The performance of the FDI scheme (summarized in Table VIII) is assessed with wind realizations which are inside the zone 3 (constant power production) of the wind turbine benchmark control scheme, i.e. wind speed in the range $12 \mathrm{~m} / \mathrm{s}-25 \mathrm{~m} / \mathrm{s}$, (see [19] for more details). The results obtained show that the FDI performance presented in this paper are not highly affected when wind velocity change within this range of wind velocities. For the control zone 2 (power optimization or partial load), i.e. wind speed in the range $3 \mathrm{~m} / \mathrm{s}-12 \mathrm{~m} / \mathrm{s}$, the pitch actuators are not active meaning that the faults regarding to this subsystem are not detectable. Moreover, a complete different set of models would be needed in order to perform FDI for the rest of the faults neglecting the pitch subsystem model. In Table VIII, the results of performing tests with smaller fault sizes with scaling and offset types of faults are shown. It is observed that the approach presented here is capable of detecting smaller faults than the nominal ones proposed in the benchmark [17], showing a good resolution of the proposed approach.

TABLE VIII

FAULT DETECTION RESUlTS

\begin{tabular}{|c|c|c|c|c|}
\hline Fault No. & $t_{D}$ required & $t_{D}$ real & Nominal Value & Minimum Fault \\
\hline$f_{1}$ & $<10 \mathrm{~T}_{s}$ & $3 \mathrm{~T}_{s}$ & 0.95 & 0.997 \\
\hline$f_{2}$ & $<10 \mathrm{~T}_{s}$ & $18 \mathrm{~T}_{s}$ & -0.5 & -0.145 \\
\hline$f_{3}$ & $<10 \mathrm{~T}_{s}$ & $3 \mathrm{~T}_{s}$ & 0.95 & 0.9999 \\
\hline$f_{4}$ & $<10 \mathrm{~T}_{s}$ & $3 \mathrm{~T}_{s}$ & - & - \\
\hline$f_{5}$ & $<10 \mathrm{~T}_{s}$ & $3 \mathrm{~T}_{s}$ & 1.1 & 1.00035 \\
\hline$f_{6}$ & $<10 \mathrm{~T}_{s}$ & $6 \mathrm{~T}_{s}$ & - & - \\
\hline$f_{7}$ & $<8 \mathrm{~T}_{s}$ & $375 \mathrm{~T}_{s}$ & - & - \\
\hline$f_{8}$ & $<100 \mathrm{~T}_{s}$ & $33 \mathrm{~T}_{s}$ & - & - \\
\hline$f_{9}$ & $<3 \mathrm{~T}_{s}$ & $3 \mathrm{~T}_{s}$ & 1000 & 25 \\
\hline$f_{10}$ & $<50 \mathrm{~T}_{s}$ & $3 \mathrm{~T}_{s}$ & - & - \\
\hline
\end{tabular}

\section{A. Isolation based on Column Reasoning}

Comparing the activated residuals in Table VII with the logic conditions described in Table $\mathrm{V}$, it is noticed that faults $f_{5}, f_{6}$ and $f_{10}$ can be isolated.

Faults $f_{4}, f_{7}$ and $f_{8}$ correspond to faults that occur in the sensors and actuators of the pitch subsystems, presenting the same fault signature. Consequently, they cannot be isolated between each other. The same occurs with faults $f_{1}$ and $f_{2}$. Faults $f_{3}$ and $f_{9}$ signatures do not match exactly with its theoretical ones because not all its residuals were activated, see Table VII. Therefore in a strict reasoning, these faults scenarios are not isolable because do not match with any of the signatures in the theoretical signature matrix. However, in the case these fault scenarios occur, it is possible to calculate which of the fault signatures is the one that adjust the best to the current observation.

\section{B. Isolation based on Row Reasoning}

Comparing the activated residuals in Table VII with the logic conditions described in Table VI, the following diagnosis results could be obtained. In the case of activation of $r_{1}$, the possible faults would be $f_{3}, f_{5}$ or $f_{9}$. In the case of $r_{3}$ or $r_{6}$ activation, the fault would be $f_{4-\beta_{1}} \vee f_{7-P A_{1}} \vee f_{8-P A_{1}}$, detecting a fault in pitch subsystem 1 but not being able to isolate whether the faulty is the sensor or the actuator. The 
same diagnosis is obtained in case that $r_{4}$ or $r_{7}$ and $r_{5}$ or $r_{8}$ activates, the fault would be in pitch subsystem 2 or 3 either in the sensor or in the actuator. If $r_{2}$ activates, $f_{9}$ would be isolated. In the case of $r_{9}$ activation, the possible faults would be $f_{3}$ or $f_{9}$. If $r_{15}, r_{16}$ or $r_{17}$ activates, a fault $f_{1-M_{B, j, m}}$ with $j=1,2,3$ or the fault $f_{2}$ could be indicated. The case of $r_{11}$ activation would result in $f_{9}$ isolation. If $r_{10}$ activates, the isolated fault would be $f_{3}$. In the case of $r_{13}$ or $r_{14}$ activation, the isolated faults would be $f_{6}$ and $f_{10}$, respectively.

\section{COMPARISON WITH OTHER APPROACHES}

Reviewing the literature, the advanced wind turbine benchmark case study used in this paper has been considered for model-based FDI in [37], [6] and [38]. The different approaches and results are briefly presented and discussed next:

- Support Vector Machines and Observers (SVMO): In [37], two methods were employed to isolate faults of different types at different locations: Support vector machines (SVM) and a Kalman-like observer. SVM could isolate most faults with the used data and characteristic vectors, except for high varying dynamics. In that case, the use of an observer, which is model-based, was found necessary. The results obtained in [37] are compared with those obtained with the approach proposed in this paper. From this comparison, it can be seen that the approach introduced here outperforms results obtained in [37].

- Support Vector Machines and Residuals Based Method (SVMR): In [6], support vector machines (SVM) and residual-based methods (RBM) are used to detect and isolate faults proposed in the benchmark. From the total set of ten faults, nine are treated in [6]. For the detection and isolation of four of the faults, SVM is employed. On the other hand, for the remaining five faults, RBM is used. The thresholds in the RBM methods proposed in [6] are generated by means of a time-variant variable function of the residuals and their mean values while the ones introduced here are adaptive thresholds determined by the effect of the uncertainty in the residuals parameters.

- Fault Detection and Isolation Filter (FDIF): the fault detection and isolation filter presented in [38] based on an optimization-based approach is proposed to deal with measurement noises in the residual generator. There are two approaches considered in [38] for the FDI filter design. The first approach does not take into account measurement noise while the second approach considers the effect of the noise in the residual, determining the transfer function and then formulating an optimization problem with the objective of minimization of the $\mathrm{H}_{2}$ norm of the calculated transfer function. The way of dealing with measurement noise in the work presented here can be related to the second approach proposed in [38] because an optimization algorithm is used to consider the variance of the noise and the approach introduced in this work uses an optimization algorithm considering the standard deviation of noise measurements. In [38], only four faults $\left(f_{3}, f_{4}, f_{7}\right.$ and $\left.f_{8}\right)$ of the total set of ten faults were considered. The fault detection times for the considered faults were not provided in [38], therefore the comparison with FDIF approach is not included in Table IX.

TABLE IX

COMPARISON OF FAULT DETECTION RESUltS

\begin{tabular}{|c|c|c|c|c|}
\hline Fault No. & $t_{D}$ required & \multicolumn{3}{|c|}{$t_{D}$ real } \\
\hline & & IBAO & SVMO & SVMR \\
\hline$f_{1}$ & $<10 \mathrm{~T}_{s}$ & $3 \mathrm{~T}_{s}$ & $\mathrm{NC}$ & $\mathrm{NC}$ \\
\hline$f_{2}$ & $<10 \mathrm{~T}_{s}$ & $18 \mathrm{~T}_{s}$ & $3 \mathrm{~T}_{s}$ & $6 \mathrm{~T}_{s}$ \\
\hline$f_{3}$ & $<10 \mathrm{~T}_{s}$ & $3 \mathrm{~T}_{s}$ & $22 \mathrm{~T}_{s}$ & $1 \mathrm{~T}_{s}$ \\
\hline$f_{4}$ & $<10 \mathrm{~T}_{s}$ & $3 \mathrm{~T}_{s}$ & $44 \mathrm{~T}_{s}$ & $6 \mathrm{~T}_{s}$ \\
\hline$f_{5}$ & $<10 \mathrm{~T}_{s}$ & $3 \mathrm{~T}_{s}$ & $11 \mathrm{~T}_{s}$ & $2 \mathrm{~T}_{s}$ \\
\hline$f_{6}$ & $<10 \mathrm{~T}_{s}$ & $6 \mathrm{~T}_{s}$ & $34 \mathrm{~T}_{s}$ & $6 \mathrm{~T}_{s}$ \\
\hline$f_{7}$ & $<8 \mathrm{~T}_{s}$ & $375 \mathrm{~T}_{s}$ & - & $2 \mathrm{~T}_{s}$ \\
\hline$f_{8}$ & $<100 \mathrm{~T}_{s}$ & $33 \mathrm{~T}_{s}$ & $12 \mathrm{~T}_{s}$ & $2 \mathrm{~T}_{s}$ \\
\hline$f_{9}$ & $<3 \mathrm{~T}_{s}$ & $3 \mathrm{~T}_{s}$ & $35 \mathrm{~T}_{s}$ & $3 \mathrm{~T}_{s}$ \\
\hline$f_{10}$ & $<50 \mathrm{~T}_{s}$ & $3 \mathrm{~T}_{s}$ & $\mathrm{NC}_{3}$ & $36 \mathrm{~T}_{s}$ \\
\hline
\end{tabular}

In the Table IX, a comparison in terms of fault detection times and the benchmark specifications is carried out for the different approaches analyzed above, where NC means not considered. The approach proposed in the present work is denoted in this table as IBAO from Interval Based ARRs and Observers. It can be seen that the IBAO approach improves the detection times for the faults $f_{4}$ and $f_{10}$. Moreover, it is the only approach from the analyzed works which considers and detects fault $f_{1}$ within the time detection requirements. Fault $f_{1}$ corresponds to a fault in the blade root moment sensor. From this result, it can be concluded that the model proposed for the blade root moment dynamics used for fault detection provided a good performance. The IBAO approach proposed in this work can detect all the fault scenarios presented in the benchmark challenge [17]. It obtains a good detection performance in the majority of them except for the fault $f_{7}$ that corresponds to an abrupt change in the pitch actuator dynamics. This is due to the fact that the observer adapts very fast to the dynamics that is tracking and consequently a considerable period of time is needed to detect fault $f_{7}$.

\section{CONCLUSIONS}

In this work, a model based diagnosis approach using interval based ARRs (static and dynamic) and observers has been applied to an advanced wind turbine benchmark, in which a set of fault scenarios was defined. In most of the cases, the obtained ARRs proved to be able to detect the different fault scenarios of different types (scaling, offset and stuck) taken into account the uncertainty in the models parameters and the noise in the sensors proposed in the benchmark. The quality of the models used for fault detection is of primary importance. In case that theoretical models do not present a good approximation of the observed dynamics, an experimental model can be used if it is correctly estimated using data.

The fault isolation techniques based on column and row reasoning applied to the signature matrix obtained from the simulation tests, have shown that only some of the faults were completely isolable. The limitation of column reasoning is that in case that not all the residuals activate, no exact match with 
theoretical FSM is obtained and therefore the isolation is not very robust. Instead, the DX row based reasoning is more robust since it allows to isolate faults even though not all the theoretical residuals are activated. As final conclusion, to improve the obtained results, it is necessary to add additional ARRs that could lead to a more complete fault signature matrix in order to improve isolation and robustness.

\section{REFERENCES}

[1] C. Walford, "Wind turbine reliability: understanding and minimizing wind turbine operation and maintenance costs," Sandia Nat. Lab., Albuquerque, New Mexico, Rep. SAND2006-1100, Mar. 2006.

[2] F. Garcia-Marquez, A. Tobias, J. Pinar-Perez, and M. Papaelias, "Condition monitoring of wind turbines: Techniques and methods," Renew. Energ., vol. 46, pp. 169-178, 2012.

[3] W. Liu, W. Zhang, J. Han, and G. Wang, "A new wind turbine fault diagnosis method based on the local mean decomposition," Renew. Energ., vol. 48, pp. 411-415, 2012.

[4] W. Yang, P. Tavner, C. Crabtree, and M. Wilkinson, "Cost-effective condition monitoring for wind turbines," IEEE Trans. Ind. Electron., vol. 57, no. 1, pp. 263-271, 2010.

[5] Z. Chen, "Fault diagnosis of gear box based on information entropy," in Proc. IEEE Control Decision Conf., Beijing, China, May 2010, pp. 1239-1242.

[6] J. Zeng, D. Lu, Y. Zhao, Z. Zhang, W. Qiao, and X. Gong, "Wind turbine fault detection and isolation using support vector machine and a residualbased method," in Proc. American Control Conf. (ACC), Washington DC, USA, 2013, pp. 3661-3666.

[7] W. Chen, S. Ding, A. Sari, A. Naik, A. Khan, and S. Yin, "Observerbased FDI schemes for wind turbine benchmark," in Proc. 18th IFAC World Congress, Milan, Italy, 2011, pp. 7073-7078.

[8] S. Karimi, A. Gaillard, P. Poure, and S. Saadate, "FPGA-based real-time power converter failure diagnosis for wind energy conversion systems," IEEE Trans. Ind. Electron., vol. 55, no. 12, pp. 4299 - 4308, 2008.

[9] S. Kia, H. Henao, and G. Capolino, "Torsional vibration effects on induction machine current and torque signatures in gearbox-based electromechanical system," IEEE Trans. Ind. Electron., vol. 56, no. 11, pp. 4689-4699, 2009.

[10] X. Gong and W. Qiao, "Bearing fault diagnosis for direct-drive wind turbines via current-demodulated signals," IEEE Trans. Ind. Electron., vol. 60, no. 8, pp. 3419-3428, 2013.

[11] H. Schulte, M. Zajac, and P. Gerland, "Takagi-Sugeno Sliding Mode Observer Design for Fault Diagnosis in Pitch Control Systems of Wind Turbines," in Proc. 8th IFAC Symp. SAFEPROCESS, Mexico City, Mexico, 2012, pp. 546-551.

[12] D. Campos-Gaona, E. Moreno-Goytia, and O. Anaya-Lara, "Fault ridethrough improvement of DFIG-WT by integrating a two-degrees-offreedom internal model control," IEEE Trans. Ind. Electron., vol. 60, no. 3, pp. 1133-1145, 2013.

[13] M. A. Parker, L. Ran, and S. J. Finney, "Distributed control of a faulttolerant modular multilevel inverter for direct-drive wind turbine grid interfacing," IEEE Trans. Ind. Electron., vol. 60, no. 2, pp. 509-522, 2013.

[14] S. Bernal-Perez, S. Ano-Villalba, R. Blasco-Gimenez, and J. RodriguezD'Derlee, "Efficiency and fault ride-through performance of a dioderectifier- and VSC-inverter-based HVDC link for offshore wind farms,' IEEE Trans. Ind. Electron., vol. 60, no. 6, pp. 2401-2409, 2013.

[15] N. Freire, J. Estima, and A. Marques Cardoso, "Open-circuit fault diagnosis in PMSG drives for wind turbine applications," IEEE Trans. Ind. Electron., vol. 60, no. 9, pp. 3957-3967, 2013.

[16] F. Vedreño Santos, M. Riera-Guasp, H. Henao, and M. Pineda-Sanchez, "Diagnosis of rotor and stator asymmetries in wound-rotor induction machines under nonstationary operation through the instantaneous frequency," IEEE Trans. Ind. Electron., vol. 61, no. 9, pp. 4947-4959, 2014.

[17] P. Odgaard and K. E. Johnson, "Wind turbine fault detection and fault tolerant control - an enhanced benchmark challenge," in Proc. American Control Conf. (ACC), Washington DC, USA, 2013, pp. 4447-4452.

[18] V. Puig, "Fault diagnosis and fault tolerant control using set-membership approaches: Application to real case studies," Int. J. Appl. Math. Comp., vol. 20, no. 4, pp. 619-635, 2010.

[19] P. Odgaard, J. Stoustrup, and M. Kinnaert, "Fault-tolerant control of wind turbines: A benchmark model," IEEE Trans. Control Syst. Technol., vol. 21, no. 4, pp. 1168-1182, 2013.
[20] J. Jonkman and L. Marshall, "Fast user's guide," Nat. Renew. Energ. Lab., Golden, Colorado, Rep. NREL/EL-500-38230, Aug. 2005.

[21] M. Blanke, M. Kinnaert, J. Lunze, and M. Staroswiecki, Diagnosis and Fault-Tolerant Control. Springer, (2nd ed.), 2006.

[22] T. van Engelen, H. Marlkou, T. Buhl, and B. Marrant, "Morphological sutdy of aerolastic control concepts for wind turbines," STABCON project, Task 7 Rep. ENK5-CT-2002-000627, Netherlands, 2007.

[23] K. Johnson, M. Pao, L. Balas, and L. Fingeresh, "Control of variablespeed wind turbines - standard and adaptive techniques for maximizing energy capture." IEEE Control Syst. Mag., vol. 26, no. 3, pp. 70-81, 2006.

[24] J. Chen and R. J. Patton, Robust Model-based Fault Diagnosis for Dynamic Systems. Kluwer Academic Publishers, (1st ed.), 1999.

[25] V. Puig, J. Quevedo, T. Escobet, F. Nejjari, and S. de las Heras, "Passive Robust Fault Detection of Dynamic Processes using Interval Models," IEEE Trans. Control Syst. Technol., vol. 16, pp. 1083-1089, 2008.

[26] J. Meseguer, V. Puig, T. Escobet, and J. Saludes, "Observer gain effect in linear interval observer-based fault detection," J. Process. Contr., vol. 20, no. 8, pp. 944-956, 2010.

[27] M. Chilali and P. Gahinet, "H $\infty$ design with pole placement constraints: an lmi approach," IEEE Trans. Autom. Control, vol. 41, no. 3, pp. 358367, 1996.

[28] V. Puig, J. Saludes, and J. Quevedo, "Worst-case simulation of discrete linear time-invariant interval dynamic systems," Reliab. Comput., vol. 9 , no. 4, pp. 251-290, 2003.

[29] T. Alamo, J. Bravo, and E. Camacho, "Guaranteed state estimation by zonotopes," Automatica, vol. 41, no. 6, pp. 1035-1043, 2005.

[30] M. Dalai, E. Weyer, and M. Campi, "Parameter identification for nonlinear systems: Guaranteed confidence regions through LSCR," Automatica, vol. 43, no. 8, pp. 1418-1425, 2007.

[31] W. Reinelt, A. Garulli, and L. Ljung, "Comparing different approaches to model error modeling in robust identification," Automatica, vol. 38, no. 5, pp. 787-803, 2002.

[32] M. Milanese, J. Norton, H. Piet-Lahanier, and E. Walter, Bounding Approaches to System Identification. Plenum Press, (1996 ed.), May 31, 1996.

[33] L. Ljung, System identification: Theory for the User. Prentice Hall, 1987.

[34] J. Gertler, Fault Detection and Diagnosis in Engineering Systems. Marcel Dekker Inc., 1998.

[35] V. Puig, A. Stancu, T. Escobet, F. Nejjari, J. Quevedo, and R. J. Patton, "Passive robust fault detection using interval observers: Application to the damadics benchmark problem." Control Eng. Pract., vol. 14, no. 6, pp. 621-633, 2006.

[36] M. Cordier, P. Dague, M. Dumas, F. Levy, J. Montmain, M. Staroswiecki, and L. Travé-Massuyès, "Conflicts versus analytical redundancy relations: A comparative analysis of the model based diagnosis approach from the articial intelligence and automatic control perspectives," IEEE Trans. Syst. Man, Cybern. B, Cybern, vol. 34, no. 5, pp. 2163-2177, 2004.

[37] N. Sheibat-Othman, S. Othman, M. Benlahrache, and P. Odgaard, "Fault detection and isolation in wind turbines using support vector machines and observers," in Proc. American Control Conf. (ACC), Washington DC, USA, 2013, pp. 4459-4464.

[38] B. Svetozarevic, M. Esfahani, M. Kamgarpour, and J. Lygeros, "Fault detection and isolation filter for a horizontal axis variable speed wind turbine," in Proc. American Control Conf. (ACC), Washington DC, USA, 2013, pp. 4453-4458.

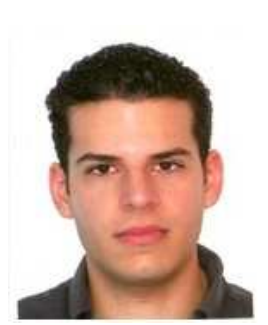

Hector Sanchez received the B.Sc. degree in Automation and Control Engineering from the University of Los Andes, Mérida, Venezuela in 2009 and the M.Sc. degree in Automation and Industrial Computing from the Universitat Politècnica de València, València, Spain in 2013. He is currently working toward a Ph.D. degree at the Research Center for Supervision, Safety and Automatic Control (CS2AC), Universitat Politècnica de Catalunya, Barcelona, Spain. He has visited the Automation \& Control Section, Aalborg University, Aalborg, Denmark from April 2014 to July 2014. His main research interests include model-based diagnosis, prognosis and the integration of control with prognosis health management focusing on wind turbines applications. 


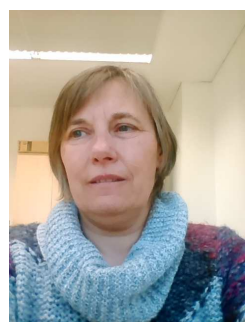

Teresa Escobet received a degree in Industrial Engineering at UPC in 1989 and $\mathrm{PhD}$ at the same University in 1997. She began to work at UPC as an Assistant Prof. in 1986 and she earned the status of Associate Prof. in 2001. Her teaching activities are related to issues in Automatic Control. She is a member of the research group Advanced Control Systems (SAC) of the Research Center for Supervision, Safety and Automatic Control (CS2AC) at UPC. Her main research interests are in dynamic system modeling and identification applied to fault detection, isolation, fault-tolerant control and condition-based maintenance. She has been involved in several International and national research projects and networks. Recent publications include 12 articles in journals and 100 papers at international conferences.

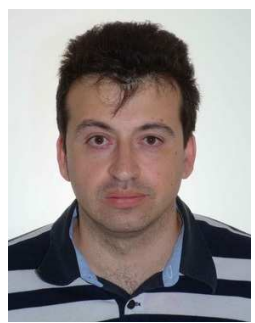

Vicenç Puig was born in Girona, Spain, in 6 November 1969. He has received the Ph.D. Degree in Control Engineering in 1999 and the Telecommunications Engineering Degree in 1993, both from the Universitat Politècnica de Catalunya (UPC), Barcelona, Spain. He is currently an Associate Professor of Automatic Control and leader of the Advanced Control Systems (SAC) research group of the Research Center for Supervision, Safety and Automatic Control (CS2AC) at UPC. His main research interests are fault detection, isolation of fault-tolerant control of dynamic systems. He has been involved in several European projects and networks and has published many papers in international conference proceedings and scientific journals.

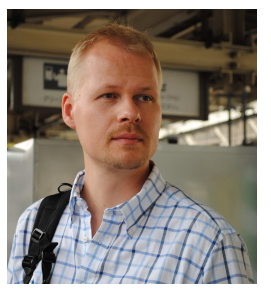

Peter Fogh Odgaard (SM'13 M'05) received the M.Sc. degree in electrical engineering and the Ph.D. degree in control engineering from Aalborg University, Aalborg, Denmark, in 2001 and 2004, respectively. He was a Guest Researcher with the Department of Mathematics, Washington University, St. Louis, MO, USA, in 2003. From September 2004 to December 2006, he was a Research Assistant Professor with Aalborg University. From January 2007 to April 2013, he was a Control Specialist with kk-electronic a/s, Denmark. In April 2013, he joined Automation \& Control, Aalborg University, as a Research Associate Professor. $\mathrm{He}$ has authored more than 70 peer-reviewed conference and journal papers, and holds four patents and patent applications. His current research interests include control of wind turbines, wind farms and renewable energy generating systems, fault detection and isolation, fault tolerant control, model predictive control and wavelets. Dr. Odgaard has been a member of IFAC Technical Committee SAFEPROCESS and Power and Energy Systems since 2011. He has served as a Co-Chair of the Danish IEEE CSS/RAS joint chapter. 